\title{
Biased attention to threat in paediatric anxiety disorders (generalized anxiety disorder, social phobia, specific phobia, separation anxiety disorder) as a function of 'distress' versus 'fear' diagnostic categorization
}

\author{
A. M. Waters ${ }^{1 *}$, B.P. Bradley ${ }^{2}$ and K. Mogg $^{2}$ \\ ${ }^{1}$ School of Applied Psychology, Griffith University, Queensland, Australia \\ ${ }^{2}$ School of Psychology, University of Southampton, UK
}

Background. Structural models of emotional disorders propose that anxiety disorders can be classified into fear and distress disorders. Sources of evidence for this distinction come from genetic, self-report and neurophysiological data from adults. The present study examined whether this distinction relates to cognitive processes, indexed by attention bias towards threat, which is thought to cause and maintain anxiety disorders.

Method. Diagnostic and attention bias data were analysed from 435 children between 5 and 13 years of age; 158 had principal fear disorder (specific phobia, social phobia or separation anxiety disorder), 75 had principal distress disorder (generalized anxiety disorder, GAD) and 202 had no psychiatric disorder. Anxious children were a clinic-based treatment-seeking sample. Attention bias was assessed on a visual-probe task with angry, neutral and happy faces.

Results. Compared to healthy controls, children with principal distress disorder (GAD) showed a significant bias towards threat relative to neutral faces whereas children with principal fear disorder showed an attention bias away from threat relative to neutral faces. Overall, children displayed an attention bias towards happy faces, irrespective of diagnostic group.

Conclusions. Our findings support the distinction between fear and distress disorders, and extend empirically derived structural models of emotional disorders to threat processing in childhood, when many anxiety disorders begin and predict lifetime impairment.

Received 11 September 2012; Revised 2 March 2013; Accepted 8 March 2013; First published online 17 April 2013

Key words: Anxiety disorders, attention bias, children, distress disorders, fear disorders.

\section{Introduction}

Childhood anxiety disorders are common, debilitating conditions associated with concurrent and long-term impairment (Bittner et al. 2007; Verduin \& Kendall, 2007), including increased risk for later psychopathology (Pine et al. 1998). Furthermore, there is high co-morbidity among childhood anxiety disorders and between anxiety disorders and other psychiatric disorders, especially depressive disorders (Kashani \& Orvaschel, 1990; Rapee et al. 2009). This co-morbidity has been explained in hierarchical models of psychiatric disorders as reflecting a general 'internalizing' factor (Kendler et al. 2003; Watson, 2005; Krueger \& Markon, 2006; Lahey et al. 2008; Seeley et al. 2011).

\footnotetext{
* Address for correspondence: Dr A. M. Waters, School of Applied Psychology, Griffith University, Queensland, 4122, Australia.

(Email: a.waters@griffith.edu.au)
}

Subsequent measurement model studies propose that the broad internalizing domain subsumes two related subfactors: 'fear' and 'distress' disorders (Clark \& Watson, 2006). In these models, specific phobia, social phobia, separation anxiety disorder, panic disorder and agoraphobia form the 'fear' disorders and generalized anxiety disorder (GAD), depression, posttraumatic stress disorder (PTSD) and dysthymia form the 'distress' disorders (Clark \& Watson, 2006).

This empirically derived structure has led to the suggestion that the classification of anxiety and depressive disorders in DSM-5 and ICD-11 should be revised into categories of fear and distress disorders (Clark \& Watson, 2006; Seeley et al. 2011). Revisions in the way anxiety disorders are conceptualized and classified have important implications for treatment and also for guiding aetiological research. Moreover, the major sources of evidence for the distinction between fear and distress disorders come from self-report, 
genetic and neurophysiological data (Kendler, 1996; Vaidyanathan et al. 2012). This highlights the importance of examining whether this distinction also relates to cognitive processes, in particular a bias in attending preferentially to threat information, which is thought to cause and maintain anxiety disorders (Williams et al. 1997; Mogg \& Bradley, 1998; Bar-Haim et al. 2007).

One widely used method of assessing attention bias is the visual-probe task with emotional words or pictures. Paired stimuli (e.g. angry face-neutral face) are briefly presented simultaneously on a computer screen followed by a visual probe in the spatial location of one of the stimuli, to which participants respond. Faster response times (RTs) to probes replacing threat compared with neutral stimuli reflect an attention bias towards threat.

In anxious adults, distress and fear disorders (e.g. GAD, social phobia) are typically associated with an attention bias towards threat (Bar-Haim et al. 2007), whereas findings from anxious children and adolescents are mixed. Several studies indicate an attention bias towards threat in children with GAD (Taghavi et al. 2003; but see Monk et al. 2006), whereas others have found a bias away from threat in childhood social anxiety (Stirling et al. 2006). Some findings suggest the bias towards threat in childhood GAD and social phobia is positively associated with higher symptom severity whereas those with anxiety disorder and low symptom severity may show no bias or threat avoidance (Waters et al. 2008a, 2012b). Yet other studies suggest that the bias towards threat is found in children with mixed anxiety disorders (i.e. GAD, social anxiety disorder, separation anxiety disorder; Hankin et al. 2010) and is not differentially affected by diagnosis type (Roy et al. 2008). However, there is a paucity of attention bias studies on varying types of specific phobias and separation anxiety disorder (In-Albon et al. 2010), and others have noted the heterogeneity of the bias in anxious youth generally (Heim-Dreger et al. 2006; Bar-Haim et al. 2011; Eldar et al. 2012). For example, less than half of children with anxiety disorders recruited into a treatment study showed a bias towards threat prior to treatment (Eldar et al. 2012).

The proposed nosological distinction between distress and fear disorders may help to explain these varied findings. Various cognitive theories of anxiety and empirical findings indicate that GAD is characterized by hypervigilance for threat (Williams et al. 1997; Mogg \& Bradley, 1998; Bar-Haim et al. 2007). Thus, attention bias towards threat may be primarily associated with distress-related anxiety disorders, which include GAD. As fear disorders differ from distress disorders in terms of avoidant responses to threat (Ollendick et al. 2002), fear disorders may be associated with attention avoidance of threat. Indeed, a recent large-scale community study indicated that children with distress disorders and high symptom severity showed an attention bias towards threat, whereas those with fear disorders and high symptom severity showed an attention bias away from threat (Salum et al. 2013). As the latter study recruited children from the community and excluded those with co-morbid diagnoses (which commonly occur in clinically anxious children), it is important to investigate whether these findings apply to clinic-based samples of treatment-seeking anxious children.

The possibility that different associations exist between the direction of threat attention bias and distress versus fear disorders has important treatment implications. For example, novel attention training treatments focus primarily on training attention away from threat (Hakamata et al. 2010). Variation in the direction of attention bias in anxious children before treatment has raised problems for the application of these treatments to childhood anxiety (Eldar et al. 2012).

In summary, examining attention bias in anxious children as a function of fear versus distress disorders is important for testing psychiatric models of anxiety disorders and may shed light on inconsistent findings of attention bias towards, versus away from, threat in anxious children. This study investigated whether the principal diagnostic category predicted threat bias in a large sample of clinically anxious and non-anxious children (aged 5-13 years). The main hypotheses were as follows: clinically anxious children with distress and fear disorders would differ in attention bias from control children; distress disorder would be associated with an attention bias towards threat relative to neutral stimuli (Hypothesis 1), whereas fear disorders would be associated with an attention bias away from threat relative to neutral stimuli (Hypothesis 2). Supplementary analyses examined whether, in comparison with control children, these predicted attention biases are more pronounced in anxious children with high symptom severity, and whether attention biases in distress and fear disorders are found for other emotional stimuli (i.e. happy faces).

\section{Method}

\section{Participants}

Participants were 453 children, aged between 5 and 13 years at screening, initially including 244 clinicreferred children and 209 control children. Attention bias data from 179 children (103 anxious children; 76 controls; $41 \%$ of sample) have been reported previously (Waters et al. 2008a, 2010, 2012a,b). Our earlier studies addressed different research questions to those 
examined here and the data have been combined to maximize the sample size.

Clinic-referred children were recruited from the Griffith University Childhood Anxiety Research Program, which provides a clinical assessment and treatment programme for children with separation anxiety disorder, social phobia, GAD and specific phobias (Waters et al. 2008b); hence, these anxiety disorders were the main focus of this study. Control children were recruited from local primary schools. Participants in the final sample satisfied the following criteria: (i) complete bias score data from the visualprobe task (bias scores were not calculated if more than $50 \%$ of RT data were missing, consistent with Roy et al. 2008; Salum et al. 2013); (ii) age between 5 and 13 years, that is the age range of children included in the treatment programme (Waters et al. 2008b); (iii) completion of diagnostic assessment, with clinic-referred children meeting criteria for a principal diagnosis of GAD, separation anxiety disorder, social phobia or specific phobia; and controls having no psychiatric disorders; and (iv) anxious children receiving no psychological or pharmacological treatment at the time of assessment.

Of the 244 clinic-referred children (who passed the initial telephone screening and completed both attention bias and diagnostic assessments), seven were excluded because of excessive missing RT data and four were excluded because they had other principal diagnoses. The final sample comprised 233 anxious children with a principal anxiety diagnosis (i.e. most severe) of GAD $(n=75)$, social phobia $(n=65)$, specific phobia $(n=75)$ or separation anxiety disorder $(n=18)$ in absence of developmental disorders, intellectual and learning disabilities, psychosis, organic brain damage or vision impairments. Disruptive disorders and depressive disorders were not reasons for exclusion as long as they were not the principal diagnosis. Co-morbidity of the final 233 anxious children was 14 with attention deficit hyperactivity disorder (ADHD), one with dysthymia, one with major depressive disorder, one with sleep terror disorder and two with enuresis.

Assignment of anxious children to 'fear' and 'distress' disorder groups was based on type of principal diagnosis (i.e. most severe, and the focus of clinical intervention). Given the co-morbidity among anxiety disorders, group allocation could not be based on exclusive diagnostic categories (see Table 1). Thus, 158 anxious children with principal specific phobia, separation anxiety disorder or social phobia were categorized into the 'fear' disorders group, and 75 children with principal GAD formed the 'distress' disorder group.

Of the initial sample of 209 controls, five were excluded because of excessive missing RT and two were excluded because they met criteria for a psychiatric disorder. The final sample was 202 children.

The study was approved by the Griffith University Human Research Ethics Committee. Parents and children provided informed consent/assent.

\section{Materials}

\section{Diagnostic assessment}

The diagnostic status of both groups was determined using the parent schedule of the Anxiety Disorders Interview Schedule for DSM-IV (ADIS-P; Silverman \& Albano, 1996). The ADIS-P contains modules for all DSM-IV anxiety disorders, depressive disorders and externalizing disorders, and screens for developmental disorders. A clinician severity rating (CSR) of $\geqslant 4$ (scale $0-8$ ) for interference caused by each diagnosis determines clinical significance. The ADIS-P was administered face-to-face or over the telephone (see Procedure). Only the parent interview schedule of the ADIS was used because of the young age of the children (Rapee et al. 1994), to avoid attrition from experimental research due to lengthy assessments and constraints on time children could be removed from class. The ADIS-P with telephone administration has good inter-rater reliability (Rapee et al. 1994), a high level of agreement with face-to-face administration (Lyneham \& Rapee, 2005), and is commonly relied upon for determining the diagnostic status of anxious and non-anxious groups (Lyneham \& Rapee, 2005, 2006). The ADIS-P was administered by postgraduate clinical students trained by clinical psychologists experienced in anxiety assessment and ADIS-P administration. Twenty per cent of interviews were audiotaped and coded by an independent rater blind to the children's diagnostic status. Inter-rater reliability showed excellent agreement (principal diagnosis $\kappa=0.85$; second diagnosis $\kappa=0.82$; third diagnosis $\kappa=0.86$ ).

\section{Anxiety symptoms}

Child and parent measures of anxiety symptoms were obtained using the Spence Children's Anxiety Scale (SCAS-C, SCAS-P; Spence, 1998; Nauta et al. 2004) The SCAS-P (39-item parent report measure) and the SCAS-C (45-item child self-report measure; six positive filler items) both contain four-point response scales $(0=$ never true to $3=$ always true), yield total scores reflecting symptom severity and possess sound psychometric properties. Mean SCAS-P total scores of 14.2 and 31.8 and mean SCAS-C total scores of 18.8 and 32.2 are reported for non-clinical and clinically anxious children respectively (Spence, 1998; Nauta et al. 2004). Cronbach's $\alpha$ in the present study was 0.81 for SCAS-P $(n=435)$ and 0.86 for SCAS-C 
Table 1. Sociodemographic, diagnostic and attention bias summary statistics as a function of diagnostic group

\begin{tabular}{|c|c|c|c|}
\hline & $\begin{array}{l}\text { Fear disorder } \\
(n=158)\end{array}$ & $\begin{array}{l}\text { Distress } \\
\text { disorder } \\
(n=75)\end{array}$ & $\begin{array}{l}\text { No disorder } \\
(n=202)\end{array}$ \\
\hline SCAS-P Total & $29.9(13.3)$ & $31.83(15.0)$ & $9.83(6.4)$ \\
\hline SCAS-C Total ${ }^{\mathrm{a}}$ & $38.8(18.0)$ & $40.0(18.3)$ & $21.5(13.3)$ \\
\hline \multicolumn{4}{|l|}{ Principal diagnosis (\%) } \\
\hline GAD & 0 & 100 & \\
\hline SAD & 11 & 0 & \\
\hline SOC & 42 & 0 & \\
\hline SP & 47 & 0 & \\
\hline Mean principal diagnosis clinician severity rating & $6.4(1.3)$ & $6.0(1.3)$ & \\
\hline Mean number of selected anxiety diagnoses & $2.3(1.0)$ & $2.3(0.9)$ & \\
\hline Mean total number of diagnoses & $2.4(1.1)$ & $2.4(1.0)$ & \\
\hline$\%$ with co-morbid disorder from the other diagnostic category & 57 & 80 & \\
\hline \multicolumn{4}{|l|}{$\%$ with co-morbid disorder as a function of anxiety diagnosis } \\
\hline Co-morbid GAD & 57 & - & \\
\hline Co-morbid SAD & - & 31 & \\
\hline Co-morbid SOC & - & 61 & \\
\hline Co-morbid SP & - & 40 & \\
\hline \multicolumn{4}{|l|}{ Parental occupational status } \\
\hline Mother & $4.5(1.1)$ & $4.7(1.1)$ & $4.5(1.2)$ \\
\hline Father & $4.3(1.1)$ & $4.2(0.9)$ & $4.2(1.1)$ \\
\hline \multicolumn{4}{|l|}{ Parental marital status } \\
\hline$\%$ married & 80 & 79 & 76 \\
\hline Age (years) & $9.1(1.9)$ & $9.4(1.6)$ & $9.1(1.7)$ \\
\hline Child's gender (M:F) & $75 / 83$ & $38 / 37$ & $96 / 106$ \\
\hline \multicolumn{4}{|l|}{ Child's country of birth } \\
\hline$\%$ Australia & 93 & 97 & 88 \\
\hline Mean RT (ms) & 595 (119) & $586(110)$ & $568(109)$ \\
\hline Mean\% trials with missing RT & $8(9)$ & $6(7)$ & $7(8)$ \\
\hline Threat bias & $-8.0(47.3)$ & $16.0(35.7)$ & $4.1(42.7)$ \\
\hline Happy bias & $7.7(44.6)$ & $5.7(45.7)$ & $4.2(42.6)$ \\
\hline
\end{tabular}

SCAS-P, Spence Children's Anxiety Scale, parent report measure; SCAS-C, Spence Children's Anxiety Scale, child self-report measure; GAD, generalized anxiety disorder; SAD, separation anxiety disorder; SOC, social phobia; SP, specific phobia; M, male; F, female; RT, response time.

Standard deviations in parentheses.

Parental occupational status determined by the Daniel Prestige Scale (Daniel, 1983). Range: 1 (high) to 7 (low).

${ }^{a}$ Sample sizes for SCAS-C total scores: Fear=125; Distress=70; Control=176.

( $n=371$, SCAS-C missing for $15 \%$ children). These anxiety measures correlated significantly $(r=0.54$, $p<0.001)$.

\section{Attention bias}

The visual-probe task was programmed using E-prime version 1.1 (Psychology Software Tools, Inc., USA) and presented on two identical Dell Optiplex computers with 17-inch 75-Hz CRT colour monitors housed in a University research laboratory and school resource room. The stimuli, used in previous studies (Roy et al. 2008), were photographs of face-pairs from 64 different actors (32 male, 32 female), each presenting a neutral and either an angry or a happy expression. This resulted in 32 angry-neutral and 32 happyneutral face-pairs. An additional 16 neutral-neutral face-pairs were used on filler trials.

\section{Procedure}

Anxious children

Participants were referred to the Griffith University Childhood Anxiety Research Program by parents, guidance counsellors, community agencies and paediatricians. The Program specializes in the treatment 
of childhood social phobia, specific phobias, GAD and separation anxiety disorder. Initial telephone screening with parents based on the ADIS-P addressed initial inclusion/exclusion criteria. Children who had probable diagnoses other than social phobia, specific phobia, separation anxiety disorder or GAD were provided with alternative treatment options and were not included further.

Parents (usually mothers) of eligible children then completed the ADIS-P with a trained postgraduate clinical student, either by telephone or during a faceto-face interview at the University. The parent and child then attended a laboratory assessment at the University, within 2 weeks of the ADIS-P assessment. Children completed the visual-probe task, and parents and children completed the SCAS, which was the primary questionnaire measure of anxiety planned for use in this study (subsamples of participants completed various additional measures for use in other projects). Eligible children were then offered cognitivebehavioural treatment (Waters et al. 2008b) and included in the present study irrespective of whether they subsequently received treatment.

\section{Control children}

These children were recruited from primary schools in response to study advertisements sent home to parents following classroom distribution of study materials or posted in school newsletters. Parents (primarily mothers) who consented to their child's participation completed the SCAS-P, and those reporting SCAS-P total scores within 1 standard deviation (S.D.) of the published norms for non-clinical children completed the ADIS-P over the telephone to confirm the absence of psychiatric diagnoses. Eligible children completed the visual-probe task and questionnaires in a resource room at their school within 2 weeks of the ADIS-P interview.

\section{Visual-probe task}

This was similar to the task used in previous studies (Roy et al. 2008). Each trial began with a 500-ms fixation point followed by two faces presented simultaneously on the left and right of the screen for $500 \mathrm{~ms}$. The face-pair was replaced with an asterisk (probe) for $1100 \mathrm{~ms}$ in the spatial location previously occupied by one of the faces. Participants pressed one of two keys labelled 'left' and 'right' as quickly as possible while avoiding mistakes, to indicate the location of the asterisk. The inter-trial interval varied randomly from 750 to $1250 \mathrm{~ms}$. The task began with 10 random practice trials, followed by one block of 80 trials. On crucial trials (i.e. angry-neutral facepairs), the probe appeared in the same spatial location as the angry face in half the trials (congruent trials), and on the opposite location to the angry face in the other half (incongruent trials). The same arrangement applied to trials with happy-neutral face-pairs. The order of trials was random for each participant.

\section{Response definitions, data screening and statistical analyses}

Analyses were conducted on attention bias scores derived from RTs to probes. Data from error trials (incorrect or no response during probe display) were excluded. Outliers were identified as RTs $<200 \mathrm{~ms}$ or $>3$ s.D. above each participant's mean, and were excluded. RTs were averaged separately for congruent and incongruent trials for each face type. Bias scores were calculated by subtracting the average RT on congruent trials from the average RT on incongruent trials. Consistent with other paediatric studies (e.g. Roy et al. 2008; Salum et al. 2013), attention bias scores were not calculated if data were missing from more than $50 \%$ of trials. Positive bias scores indicate attention towards angry relative to neutral faces; negative values indicate attention away from angry faces.

Hypotheses concerning attention bias for angry faces were tested using an ANOVA with diagnostic group (distress, fear, no disorder) as the between-subjects independent variable. Follow-up tests used planned group comparisons with disorder-free children as the referent (control) group and one-sample $t$ tests comparing bias scores against zero. Partial eta squared $\left(\eta_{\mathrm{p}}^{2}\right)$ was calculated as an estimate of effect size. Supplementary analyses examined whether previously reported data (Waters et al. 2008a, 2010, 2012a,b) differed from new data by including the data set (old, new) as an independent variable. The effect of anxiety symptom (AS) severity on threat bias was examined using a median split on SCAS-P scores (the median score of children with anxiety disorders was 29) to allow comparison with prior research using a similar dichotomous split on symptom severity (e.g. Waters et al. 2008a; Salum et al. 2013). This produced five groups: (i) distress disorder with low AS, $n=37$; (ii) distress disorder with high AS, $n=38$; (iii) fear disorder with low AS, $n=81$; (ii) fear disorder with high AS, $n=77$; and (v) no disorder with low AS, $n=200$ (only two control children had SCAS-P scores $>29$ and were excluded from these analyses). Similar tests were used for happy face bias scores.

\section{Results}

\section{Group characteristics}

Group comparisons showed a significant main effect of diagnostic group (distress, fear, no disorder) on 


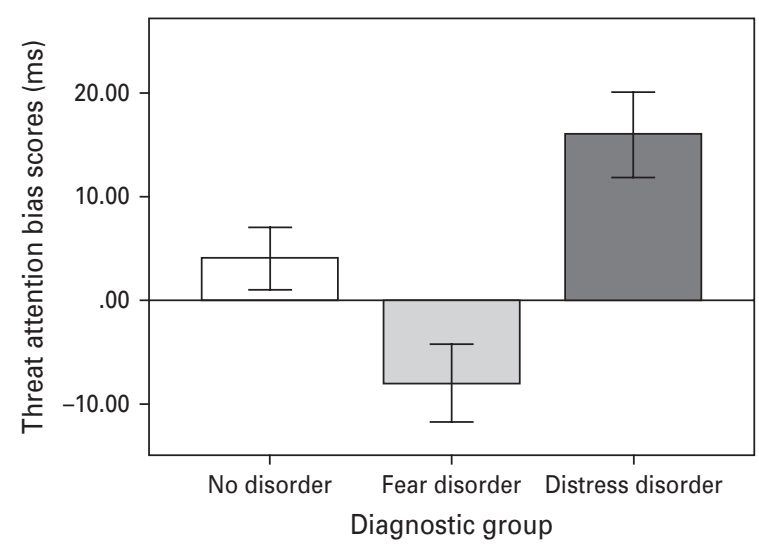

Fig. 1. Mean attention bias scores (in ms; error bars indicate \pm 1 s.E.) for angry faces relative to neutral faces as a function of diagnostic group.

SCAS-P and SCAS-C total scores: $F_{2,432}=191.16$, $p<0.001, \eta_{\mathrm{p}}^{2}=0.47$ and $F_{2,368}=56.87, p<0.001, \eta_{\mathrm{p}}^{2}=0.24$ respectively (see Table 1 for details). Fear and distress disorder groups had significantly higher SCAS-P and SCAS-C total scores compared with controls (all $p^{\prime}$ s <0.001), whereas the two diagnostic groups did not differ significantly on either measure (both $p^{\prime}$ s >0.97). The fear disorder group had a higher CSR of principal diagnosis than the distress disorder group $\left(F_{1,231}=4.70, p=0.031, \eta_{\mathrm{p}}^{2}=0.02\right)$. Groups did not differ significantly in the number of co-morbid anxiety or other diagnoses (both $F^{\prime} \mathrm{s}<1$ ).

$\chi^{2}$ analyses showed no significant diagnostic group differences in child gender ratio, child country of birth (Australian, other), parents' marital status (married, other) or occupational prestige. There were no significant diagnostic group differences in age, overall mean RT and percentage of missing data.

\section{Attention bias for angry faces}

The ANOVA showed a significant main effect of diagnostic group $\left(F_{2,432}=8.35, p<0.001, \eta_{\mathrm{p}}^{2}=0.04\right)$ (see Fig. 1). This was unaffected by data set (old, new) $(F<1)$. Planned group comparisons showed that the distress and fear disorder groups each differed significantly in threat bias scores from the controls ( $p=0.043$ and 0.009 respectively). One-sample $t$ tests confirmed that children with principal distress disorder displayed a significant bias towards threat compared with neutral faces $\left(t_{74}=3.89, p<0.001\right)$ (consistent with Hypothesis 1); children with a principal fear disorder displayed a significant bias away from threat relative to neutral faces $\left(t_{157}=2.13, p=0.035\right)$ (consistent with Hypothesis 2); disorder-free children showed no significant bias $\left(t_{201}=1.37, p=0.17\right)$.
Table 2. Mean anxiety (SCAS-P) and attention bias scores as a function of diagnostic category and anxiety symptom (AS) severity

\begin{tabular}{|c|c|c|c|c|c|}
\hline & \multirow[b]{2}{*}{$\begin{array}{l}\text { No } \\
\text { disorder } \\
\text { Low AS } \\
(n=200)\end{array}$} & \multicolumn{2}{|c|}{ Fear disorder } & \multicolumn{2}{|c|}{ Distress disorder } \\
\hline & & $\begin{array}{l}\text { Low } \\
\text { AS } \\
(n=81)\end{array}$ & $\begin{array}{l}\text { High } \\
\text { AS } \\
(n=77)\end{array}$ & $\begin{array}{l}\text { Low } \\
\text { AS } \\
(n=37)\end{array}$ & $\begin{array}{l}\text { High } \\
\text { AS } \\
(n=38)\end{array}$ \\
\hline $\begin{array}{c}\text { SCAS-P } \\
\text { Total }\end{array}$ & $10(6)$ & $19(7)$ & $41(8)$ & $21(6)$ & $43(13)$ \\
\hline Threat bias & $4(42)$ & $-5(45)$ & $-11(50)$ & $11(36)$ & $21(35)$ \\
\hline Happy bias & $4(43)$ & $16(45)$ & $-2(42)$ & $3(34)$ & $8(55)$ \\
\hline
\end{tabular}

SCAS-P, Spence Children's Anxiety Scale, parent report measure.

Standard deviations in parentheses.

\section{Supplementary analyses of threat bias scores}

ANOVA comparing the four principal anxiety diagnoses showed a significant main effect of diagnosis on threat bias scores $\left(F_{3,229}=5.78, p<0.01, \eta_{\mathrm{p}}^{2}=0.07\right)$. Post-hoc contrasts showed no significant differences between the three fear disorders, whereas each fear disorder differed significantly in threat bias from GAD [mean bias (s.D.): principal social phobia, -11 (44); specific phobia, -3 (49); separation anxiety disorder, -19 (54); GAD 16 (36)].

As the fear and distress disorder groups differed in CSR of principal diagnosis, their threat attention bias scores were entered into an ANCOVA with ADIS-P CSR as the covariate and diagnostic group as the between-subjects independent variable. There was no main or interacting effect of the covariate; the main effect of the diagnostic group remained significant $\left(F_{1,229}=6.07, p=0.01, \eta_{\mathrm{p}}^{2}=0.03\right)$.

We examined effects of diagnostic group and AS level on threat bias, as described earlier (Table 2). The five-way ANOVA showed a significant main effect of subgroup $\left(F_{4,428}=4.63, p=0.001, \eta_{\mathrm{p}}^{2}=0.04\right)$ (Fig. 2). This effect was independent of data set (old, new). Planned comparisons using controls as the referent group (cf. Salum et al. 2013) showed that they differed significantly in threat bias from children with distress disorder and high AS $(p=0.02)$ and from those with fear disorder and high AS $(p=0.01)$, but not from those with distress disorder and low AS $(p=0.38)$ or fear disorder and low AS $(p=0.13)$. One-sample $t$ tests showed a significant attention bias towards threat in distress disorder and high AS $\left(t_{37}=3.72\right.$, $p<0.001)$ and a bias away from threat in fear disorder and high AS $\left(t_{76}=1.96, p=0.05\right)$, whereas biases in the other subgroups were not significantly different from zero (distress disorder and low AS: $t_{36}=1.80, p=0.08$; fear disorder and low AS: $t_{80}=1.00, p=0.32$; no 


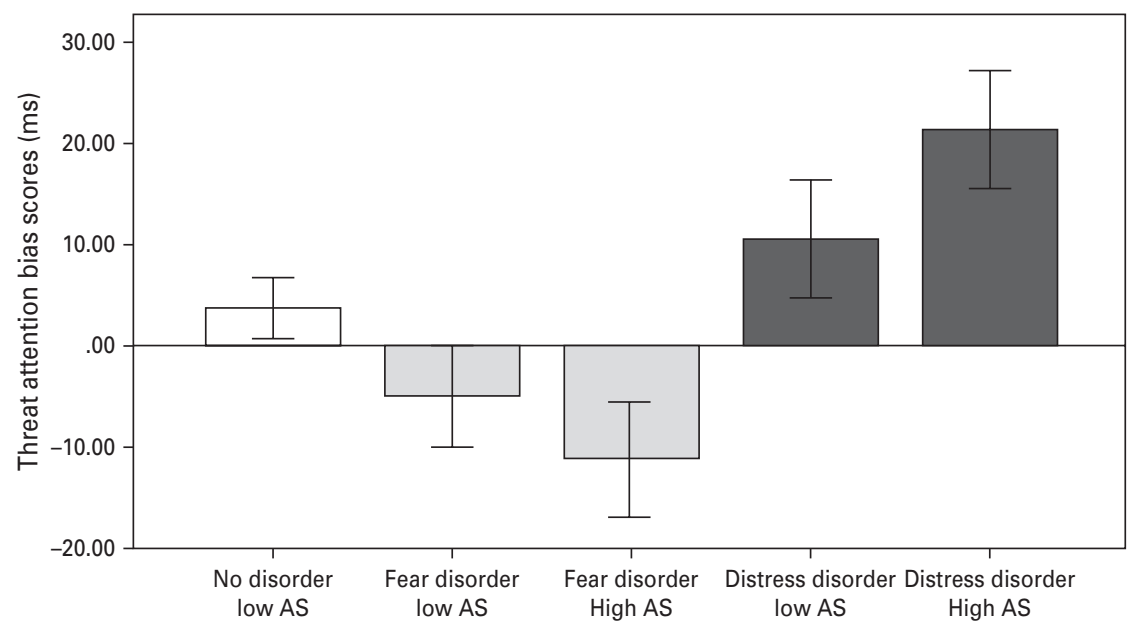

Fig. 2. Mean attention bias scores (in ms; error bars indicate \pm 1 s.e.) for angry faces relative to neutral faces as a function of diagnostic group and anxiety symptom (AS) severity.

disorder: $t_{199}=1.23, p=0.22$ ). Within each disorder group, threat bias was not correlated significantly with SCAS-P scores.

\section{Attention bias for happy faces}

The three-way ANOVA of attention bias scores for happy faces found no significant effect of diagnostic group $(F<1)$ (see Table 1$)$. Overall, children showed a significant bias towards happy relative to neutral faces, that is one-sample $t$ test contrasting bias score against zero $\left(t_{434}=2.71, p=0.007\right.$; mean $=6 \mathrm{~ms}$, S.D. $=44)$. Supplementary analyses showed no significant effect of type of diagnosis within the fear disorder group. The five-way ANOVA assessing the effect of diagnostic group and AS severity showed no significant results (see Table 2).

\section{Discussion}

Consistent with hypotheses, the present study found that, in comparison with healthy controls, children with principal distress disorder (i.e. GAD) displayed an attention bias towards angry relative to neutral faces, whereas children with principal fear disorders showed an attention bias away from angry relative to neutral faces. There were no significant group differences in attention to positive stimuli; overall, children showed a significant bias towards happy relative to neutral faces.

The present findings add to growing evidence across self-report, genetic and neurophysiological data for distinctions between fear and distress disorders (Kendler, 1996; Vaidyanathan et al. 2012). This study found that the distinction between fear- and distress-related anxiety disorders manifests early in development in cognitive processes such as attention bias, as children with principal fear disorders avoid threat stimuli and those with principal distress disorder orient towards them. Attention towards threat in children with distress disorder is consistent with some prior findings in children (Taghavi et al. 2003; Waters et al. 2008a) and adults with GAD (Bradley et al. 1999; Bar-Haim et al. 2007). Attention bias studies of children with fear disorders are sparse; however, attention avoidance of threat has been reported in children with social anxiety (Stirling et al. 2006) and with fear disorders as a group (Salum et al. 2013). Attention avoidance of threat may be associated with behavioural avoidance that often distinguishes fear disorders from other anxiety disorders in children (Eldar et al. 2012). Attention avoidance may also reflect a cognitive strategy following an initial vigilance response that occurs too rapidly to be detected with the paradigm used here (Salum et al. 2013), which gives a 'snapshot' measure of attention bias (500-ms post-stimulus onset). Future research should examine the time course of attention bias in anxiety disorders across development; that is whether fear disorders are associated with initial orienting towards threat followed by avoidance whereas distress disorders are associated with initial orienting towards threat followed by continued threat monitoring, and whether attention avoidance of threat is more apparent in fearful children than adults, perhaps due to poorer attention control in childhood.

As noted earlier, some previous research suggested that attention biases in children also vary as a function of symptom severity (Waters et al. 2008a, 2010, 2012b; Salum et al. 2013). In the latter large-scale community study, children with distress disorders and high symptom severity showed increased attention bias towards threat whereas those with fear disorders and high symptom severity showed avoidance of threat, relative 
to low-symptom disorder-free children (Salum et al. 2013). Supplementary analyses in the present study showed a similar pattern of results: relative to lowanxious controls, children with principal distress disorder and high AS severity showed greater attention bias towards threat whereas those with fear disorder and high symptom severity showed greater bias away from threat. The present results also concur with our earlier report of greater bias towards threat in children with distress disorder and high AS severity relative to disorder-free children (Waters et al. 2008a) (31\% of the present distress disorder group included this earlier sample, that is 23 of 75; with no significant difference in bias between old and new samples). The results do not concur with Waters et al. (2012b), which suggested a bias towards threat in children with principal social anxiety disorder and high AS severity; these children had high GAD co-morbidity and the sample size was small $(n=13)$. In comparison, the size of the subgroup with fear disorder and high AS severity was considerably larger in the present study $(n=77)$. Given some inconsistencies in findings, the results from the supplementary analyses, which include symptom severity as an additional independent variable, should be interpreted with caution. Nevertheless, they suggest that, although principal diagnostic category is a key determinant of the direction of threat attention bias, it is also useful to assess effects of anxiety severity, as it may help to explain mixed findings across studies. For example, in both this study and that of Salum et al. (2013), children with anxiety disorders and low symptom severity did not show significant attention bias for threat, compared with low-anxious disorder-free children, which suggests that studies using small samples of children with assorted principal anxiety disorders of low severity may have difficulty in detecting attention biases.

Findings from the primary analyses have important clinical implications. The direction of attention bias has been shown to predict treatment outcome in anxious adults and children; that is avoidance of angry faces pre-treatment predicted poorer outcome (Price et al. 2011; Waters et al. 2012a). Moreover, novel attention training interventions, which train anxious individuals to be threat avoidant, have shown promising effects in anxious adults (Hakamata et al. 2010). However, this approach may be unsuitable for anxious individuals who show attention avoidance of threat prior to treatment (Eldar et al. 2012). Thus, the application of such treatments to children would benefit from clarification of the mechanisms underlying attention bias in childhood anxiety disorders.

Although this study has several strengths, including a large sample of clinically anxious and non-anxious children $(n=435)$ and reliance on well-established diagnostic, symptom and attention bias measures, there are limitations. One limitation is reliance on the parent interview schedule of the ADIS to confirm children's diagnoses. This study also did not include children with fear and distress disorders other than social phobia, GAD, specific phobia and separation anxiety disorder, thus it examines the fear/distress distinction within anxiety, rather than emotional disorders in general. Finally, although it is informative to compare children with single diagnoses (Salum et al. 2013), this was not feasible because of the high co-morbidity. Thus, the present study complements and extends previous research because findings from children with single diagnoses cannot be assumed to generalize to clinic-based treatment-seeking anxious children who have high rates of co-morbidity (Rapee et al. 2009). The approach used here facilitates comparison with other childhood anxiety studies, which commonly select children based on their principal diagnosis. Moreover, differences in threat attention biases were found between principal fear versus distress disorders, despite co-morbidity on subsidiary diagnoses, indicating that the relationship between type of principal disorder and attention bias deserves further investigation.

In summary, the present results add to growing evidence for the distinction between fear and distress disorders and extend this research to treatment-seeking anxious children. Children with principal fear disorders showed greater attention avoidance of threat stimuli, and children with a principal distress disorder (GAD) showed greater attention bias towards threat stimuli, in comparison with healthy controls. Studies including adolescents and adults are required so that the relationship between threat attention bias and fear versus distress disorders can be tracked throughout development.

\section{Acknowledgements}

This research was supported by Australian Research Council Grant DP1095536. We thank N. Stoilov for assistance with data collection and D. S. Pine for helpful feedback on an earlier draft.

\section{Declaration of Interest}

None.

\section{References}

Bar-Haim Y, Lamy D, Pergamin L, Bakermans-Kranenburg MJ, van IJzendoorn MH (2007). Threat-related attentional bias in anxious and nonanxious individuals: a meta-analytic study. Psychological Bulletin 133, 1-24.

Bar-Haim Y, Morag I, Glickman S (2011). Training anxious children to disengage attention from threat: a randomized 
controlled trial. Journal of Child Psychology and Psychiatry, and Allied Disciplines 52, 861-869.

Bittner A, Egger HL, Erkanli A, Costello J, Foley DL, Angod A (2007). What do childhood anxiety disorders predict? Journal of Child Psychology and Psychiatry, and Allied Disciplines 48, 1174-1183.

Bradley BP, Mogg K, White J, Groom C, de Bono J (1999). Attentional bias for emotional faces in generalized anxiety disorder. British Journal of Clinical Psychology 38, 267-278.

Clark LA, Watson D (2006). Distress and fear disorders: an alternative empirically based taxonomy of the 'mood' and 'anxiety' disorders. British Journal of Psychiatry 189, 481-483.

Daniel AE (1983). Power, Privilege and Prestige. Longman Cheshire: Melbourne.

Eldar S, Apter A, Lotan D, Perez-Edgar K, Naim R, Fox NA, Pine DS, Bar-Haim Y (2012). Attention bias modification treatment for pediatric anxiety disorders: a randomized control trial. American Journal of Psychiatry 169, 213-230.

Hakamata Y, Lissek S, Bar-Haim Y, Britton JC, Fox N, Leibenluft E, Ernst M, Pine DS (2010). Attention bias modification treatment: a meta-analysis towards the establishment of novel treatment for anxiety. Biological Psychiatry 68, 982-990.

Hankin BL, Gibb BE, Abela JRZ, Flory K (2010). Selective attention to affective stimuli and clinical depression among youths: role of anxiety and specificity of emotion. Journal of Abnormal Psychology 119, 491-501.

Heim-Dreger U, Kohlmann CW, Eschenbeck H, Burkhardt U (2006). Attentional biases for threatening faces in children: vigilant and avoidant processes. Emotion 6, 320-325.

In-Albon T, Kossowsky J, Schneider S (2010). Vigilance and avoidance of threat in eye movements of children with separation anxiety disorder. Journal of Abnormal Child Psychology 38, 225-235.

Kashani JH, Orvaschel H (1990). A community study of anxiety in children and adolescents. American Journal of Psychiatry 147, 313-318.

Kendler KS (1996). Major depression and generalised anxiety disorder: same genes, (partly) different environments revisited. British Journal of Psychiatry 168 (Suppl. 30), 68-75.

Kendler KS, Prescott CA, Myers J, Neale MC (2003). The structure of genetic and environmental risk factors for common psychiatric and substance use disorders in men and women. Archives of General Psychiatry 60, 929-937.

Krueger RF, Markon KE (2006). Reinterpreting comorbidity: a model-based approach to understanding and classifying psychopathology. Annual Review of Clinical Psychology 2, 111-133.

Lahey BB, Rathouz PJ, Van Hulle C, Urbano RC, Krueger RF, Applegate B, Garriock HA, Chapman DA, Waldman ID (2008). Testing structural models of DSM-IV symptoms of common forms of child and adolescent psychopathology. Journal of Abnormal Child Psychology 36, 187-206.

Lyneham HJ, Rapee RM (2005). Agreement between telephone and in-person delivery of a structured interview for anxiety disorders in children. Journal of the American Academy of Child and Adolescent Psychiatry 44, 274-282.
Lyneham HJ, Rapee RM (2006). Evaluation of therapist-supported parent- implemented CBT for anxiety disorders in rural children. Behaviour Research and Therapy 44, 1287-1300.

Mogg K, Bradley BP (1998). A cognitive-motivational analysis of anxiety. Behaviour Research and Therapy 36, 809-848.

Monk CS, Nelson EE, McClure EB, Mogg K, Bradley BP, Leibenluft E, Blair RJ, Chen G, Charney DS, Ernst M, Pine DS (2006). Ventrolateral prefrontal cortex activation and attentional bias in response to angry faces in adolescents with generalized anxiety disorder. American Journal of Psychiatry 163, 1091-1097.

Nauta MH, Scholing A, Rapee R, Abbott M, Spence SH, Waters AM (2004). Parent-report measure of children's anxiety: psychometric properties and comparison with child-report in clinical and normal samples. Behaviour Research and Therapy 42, 413-439.

Ollendick TH, King NJ, Muris P (2002). Fears and phobias in children: phenomenology, epidemiology, and aetiology. Child and Adolescent Mental Health 7, 98-106.

Pine DS, Cohen P, Gurley D, Brook J, Ma Y (1998). The risk for early-adulthood anxiety and depressive disorders in adolescents with anxiety and depressive disorders. Archives of General Psychiatry 55, 56-64.

Price M, Tone EB, Anderson PL (2011). Vigilant and avoidant attention biases as predictors of response to cognitive behavioural therapy for social phobia. Depression and Anxiety 28, 349-353.

Rapee RM, Barrett P, Dadds M, Evans L (1994). Reliability of DSM-III-R child anxiety disorders using structured interview: inter-rater and parent-child agreement. Journal of the American Academy of Child and Adolescent Psychiatry 33, 984-992.

Rapee RM, Schniering C, Hudson JL (2009). Anxiety disorders during childhood and adolescents: origins and treatment. Annual Review of Clinical Psychology 5, 311-341.

Roy AK, Vasa RA, Bruck M, Mogg K, Bradley BP, Sweeney M, Bergman RL, McClure-Tone EB, Pine DS; CAMS Team (2008). Attention bias toward threat in paediatric anxiety disorders. Journal of the American Academy of Child and Adolescent Psychiatry 47, 1189-1196.

Salum GA, Mogg K, Bradley BP, Gadelha A, Pan P, Tamanaha AC, Moriyama T, Graeff-Martins AS, Jarros RB, Polanczyk G, do Rosário MC, Leibenluft E, Rohde LA, Manfro GG, Pine DS (2013). Threat bias in attention orienting: evidence of specificity in a large community-based study. Psychological Medicine 43, 733-745.

Seeley JR, Kosty DB, Farmer RF, Lewinsohn PM (2011). The modeling of internalizing disorders on the basis of patterns of lifetime comorbidity. Journal of Abnormal Psychology 120, 308-321.

Silverman WK, Albano AM (1996). Anxiety Disorders Interview Schedule for DSM-IV: Child and Parent Versions. The Psychological Corporation: San Antonio, TX.

Spence SH (1998). A measure of anxiety symptoms among children. Behaviour Research and Therapy 36, 545-566.

Stirling LJ, Eley TC, Clark DM (2006). Preliminary evidence for an association between social anxiety 
symptoms and avoidance of negative faces in school-age children. Journal of Clinical Child and Adolescent Psychology 35, 440-445.

Taghavi MR, Dalgleish T, Moradi AR, Neshat-Doost HT, Yule W (2003). Selective processing of negative emotional information in children and adolescents with generalized anxiety disorder. British Journal of Clinical Psychology 42, 221-230.

Vaidyanathan U, Nelson LD, Patrick CJ (2012). Clarifying domains of internalizing psychopathology using neurophysiology. Psychological Medicine 42, 447-459.

Verduin TL, Kendall P (2007). Peer perceptions and liking of children with anxiety disorders. Journal of Abnormal Child Psychology 36, 459-469.

Waters AM, Henry J, Mogg K, Bradley BP, Pine DS (2010). Attentional bias towards angry faces in childhood anxiety disorders. Journal of Behavior Therapy and Experimental Psychiatry 41, 158-164.

Waters AM, Mogg K, Bradley BP (2012a). The direction of threat attention bias predicts treatment outcome from cognitive behavioural therapy in anxious children. Behaviour Research and Therapy 50, 428-424.

Waters AM, Mogg K, Bradley BP, Pine DS (2008a). Attentional bias for emotional faces in children with generalized anxiety disorder. Journal of the American Academy of Child and Adolescent Psychiatry 47, 435-442.

Waters AM, Mogg K, Bradley BP, Pine DS (2012b). Attention bias for angry faces in children with social phobia. Journal of Experimental Psychopathology 2, 475-489.

Waters AM, Wharton TA, Zimmer-Gembeck MJ,

Craske MG (2008b). Threat-based cognitive biases in anxious children: comparison with non-anxious children before and after cognitive behaviour treatment. Behaviour Research and Therapy 46, 358-406.

Watson D (2005). Rethinking mood and anxiety disorders: a quantitative hierarchical model for DSM-V. Journal of Abnormal Psychology 114, 522-536.

Williams JMG, Watts FN, MacLeod C, Mathews A (1997). Cognitive Psychology and Emotional Disorders, 2nd edn. Wiley: Chichester. 